\title{
Gingival and bone healing patterns with ridge preservation in beagle dogs
}

\author{
Xing-Hui Piao ${ }^{1 \dagger}$, Hyo-Seon Park ${ }^{2 \dagger}$, Eui-Ri Na ${ }^{3}$, Young-Joon Kim4, and Jong-Wook Moon ${ }^{2 *}$ \\ ${ }^{1}$ Doctor, Department of Dentistry, Affiliated Hospital of Yanbian University, Yanji, Jilin, China \\ ${ }^{2}$ Master's Degree, Department of Periodontology, School of Dentistry, Chonnam National University, Gwangju, Republic of Korea \\ ${ }^{3}$ Doctor's Degree, Department of Periodontology, School of Dentistry, Chonnam National University, Gwangju, Republic of Korea \\ ${ }^{4}$ Professor, Department of Periodontology, School of Dentistry, Chonnam National University, Gwangju, Republic of Korea
}

\begin{abstract}
The aim of this study was to evaluate the clinical and histologic findings of bone and gingival healing patterns with ridge preservation. The mandibular third premolars were extracted from beagle dogs and the dogs were euthanized after 12 weeks. The ridge preservation was performed and the animals were divided into 3 groups, including 1) Control: extraction; 2) surederm group: extraction+acellular dermal matrix (Surederm); 3) collatape group: extraction+collagen membrane (Collatape). Changes in the keratinized gingiva and bone volume of the extraction sockets were measured. In the surederm and collatape group, the decrease in keratinization was small and statistically insignificant. The surederm group had significantly greater dimensions of buccal gingiva as compared to the control and collatape groups at 4 weeks. The surederm group had greater dimensions of buccal gingiva as compared to the control group at 8 weeks. Histological observation showed that in the surederm group, a dark red-colored osteoid seam was observed around the alveolar bone. Osteoid seams were observed directly below and to the side of the socket. In the collatape group, a connective tissue band was observed at the site of contact with the alveolar bone. Both experimental groups had more alveolar bone volume than the control. The alveolar bone volume in the surederm group was significantly greater than that of the collatape group. The results suggest that the acellular dermal matrix results in better healing patterns than other methods.
\end{abstract}

Key Words: Acellular dermal graft tissue, Animal, Collagen, Models, Tooth socket

(c) This is an open-access article distributed under the terms of the Creative Commons Attribution Non-Commercial License (http://creativecommons.org/licenses/by-nc/4.0) which permits unrestricted noncommercial use, distribution, and reproduction in any medium, provided the original work is properly cited.

\section{Introduction}

The alveolar ridge begins to absorb on healing process after tooth extraction. After extraction, about 40\%-60\% of the alveolar bone is reduced in 6-month. Especially the buccal alveolar ridge exhibits more absorption compared to the lingual side $[1,2]$. Even if the use of ridge preservation, it could not completely stop the alveolar ridge ab- sorption. Giorgio et al. [3] also concluded that it couldn' t completely prevent the buccal alveolar ridge absorption. Furthermore, although the bone grafting materials in the extraction socket can effectively save the volume, it didn't promote the formation of new bone, but delayed the bone healing reaction $[4,5]$. As the alveolar bone was absorbed, the amount and width of the gingiva, especially keratinized gingiva, decreases. Therefore, when a certain period of

Received February 17, 2020; Revised [1] April 17, 2020; [2] June 1, 2020; Accepted June 5, 2020

${ }^{\star}$ Corresponding author: Jong-Wook Moon, Department of Periodontology, School of Dentistry, Chonnam National University, 33 Yongbongro, Buk-gu, Gwangju 61186, Republic of Korea.

Tel: +82-62-530-5648, Fax: +82-62-530-5649, E-mail: cuidezhe@empal.com

${ }^{\dagger}$ These authors contributed equally to this work.

Copyright $\odot$ 2020, Oral Biology Research Institute 
time passed after tooth extraction, there were decreasing not only the width and height of the alveolar bone, but also the reduction of the upper soft tissue width, especially the keratinized gingiva. In this situation, the amount of keratinized gingiva as well as the amount and height of the alveolar bone are insufficient when the implant is planned at the site. And also even if the implant was successfully placed in the alveolar bone, it is difficult to control the oral hygiene and may affect the long-term maintenance of the implant.

After tooth extraction, when immediately apply the ridge preservation surgery, because of the lack of mucosa, it is difficult to suture to cover the materials and the membrane, resulting in the membrane exposure during healing. In order to solve this problem, the free gingival graft is planned to achieve full coverage of gingiva [6]. However, for free gingival graft, a secondary surgical site is required and there is a limit to the amount to be obtained. Recently, it has been reported that acellular dermal matrix (Alloderm; LifeCell Co., The Woodlands, TX, USA) can play as a role in increase of the attached gingiva and as an resorbable membrane [7-19]. It has been reported in several clinical cases that the ridge preservation with the using of acellular dermal matrix as a membrane to cover the bone graft materials or to the augment of already absorbed ridge [16,20].

However, there are no well-documented histologic reports on the healing patterns of gingival tissues and healing changes in alveolar ridge. There are many ridge preservation techniques after extraction. Many studies have focused on the preservation of the alveolar bone in the extraction socket, and few studies have report for the preservation and healing patterns of soft tissue on top of the alveolar bone. Therefore, the purpose of this study is to evaluate clinical and histologic findings of gingival changes and how it affects healing pattern after tooth extraction when performed the ridge preservation with acellular dermal matrix and collagen membrane.

\section{Materials and Methods}

\section{Animal}

The animal ethics committee of Chonnam National University was approved (CNU IACUC-YB-2017-21). Ten male beagle dogs (age: 12-16 months; weight: 10-12 kg) were used in this study. To make the same oral environment before the experiment, we performed scaling and plaque removal. Thereafter, oral gel containing chlorhexidine was used for 2 weeks to maintain healthy periodontal status. In beagle dogs, bilateral mandibular third premolars were extracted and randomly divided into 3 groups: 1) The control group extracted only the mandibular third premolar; 2) The surederm group extracted the mandibular third premolar and treated the socket with acellular dermal matrix (Surederm; Hans Biomed, Seoul, Korea); 3) The collatape group extracted the mandibular third premolar and treated the socket with collagen membrane (Collatape; Integra Lifesciences Corp., Plainsboro, NJ, USA).

\section{Surgical procedure}

Beagle dogs were anesthetized with $10 \mathrm{mg} / \mathrm{kg}$ ketamine (Yuhan corp., Seoul, Korea) before the surgery. Atraumatic extraction was performed on the mandibular third premolar of the beagle dog, and washed three times with saline solution. In the control group was extracted and was sutured with 3-0 silk (Mersilk; Ethicon Ltd., Edinburgh, UK). In surederm group, after extraction, 2-3 mm circular internal bevel incision and full-thickness flap elevation was done, and then extraction socket was covered with acellular dermal matrix (Surederm). And all materials were completely covered with flap. And interrupted suture was done with 3-0 silk. In the collatape group, after extraction, the surrounding area was adjusted with the same as the surederm group, and then extraction socket was covered with collagen membrane (Collatape) and interrupted suture was done with 3-0 silk. The suture was removed after 10 days.

After the surgeries, the beagle dogs were given antibiotics (Amoxapen Cap, 20 mg/kg amoxicillin; Chong Kun Dang Pharmaceutical Corp., Seoul, Korea) twice daily for 7 days. Anti-inflammatory and analgesic drugs $5 \mathrm{mg} / \mathrm{kg}$ firocoxib (Previcox 227 mg; Merial Ltd., Duluth, GA, USA) was administered orally once a day for 7 days. The beagle dog' s mouth was irrigated with $0.12 \%$ chlorhexidine gluconate solution (Hexamedine; Bukwang, Seoul, Korea). The beagle dogs were kept in kennels and on concrete runs at the university's field laboratory with free access to water and feed 
of moistened balanced dog's chow.

A daily inspection of the wounds for clinical signs of complications. The beagle dogs were sacrificed 12 weeks after the surgery applying an overdose of Thiopental (SK Chemicals Co., Ltd., Gunpo, Korea).

\section{Gingival examination}

The width of gingiva was measured in millimeters by a periodontal probe (Williams probe; Hu-friedy, Chicago, IL, USA) in the buccal side and the alveolar crest (buccolingual direction) at 2, 4, 6, 8 weeks after the surgery and sacrifice (12 weeks postoperatively). The gingival width in the alveolar crest was the distance from the lingual to the buccal alveolar ridge. The gingival width in the buccal side was the distance from the buccal alveolar ridge to the mucogingival junction (Fig. 1).

\section{Histological examination}

Twelve weeks after the surgery, the animals were euthanized with anesthetic overdose and mandible were removed in block, being immediately immersed in buffered 10\% formalin (Junsei Chemical Co., Tokyo, Japan) for 48 hours. The tissue specimens were fixed in the buffered

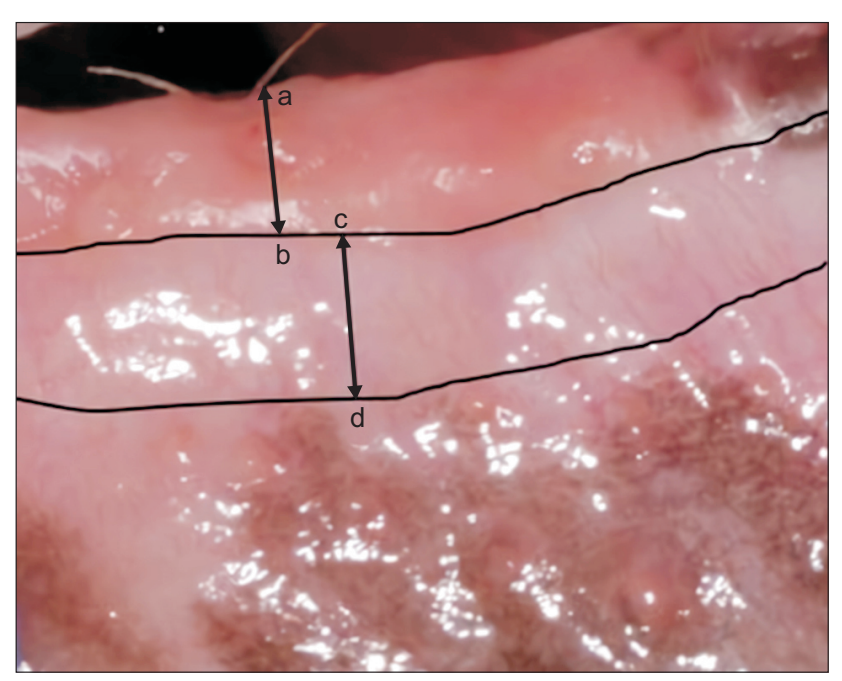

Fig. 1. The gingival measurements of the buccal side and the alveolar crest. (a) Lingual alveolar ridge; (b, c) buccal alveolar ridge; (d) mucogingival junction; $(a, b)$ width of gingiva at alveolar crest; (c, d ) width of gingiva at buccal side. neutral formalin (Sigma Aldrich) solution for 2 weeks, and the tissue specimens were dehydrated with increasing ethanol concentration. When dehydration was completed, the tissues were perfused with increasing the resin ratio with a mixture of ethanol and Technovit 7200 resin (Heraeus Kulzer, Wehrheim, Germany). After the infiltration, the tissues were embedding which UV embedding system (KULZER EXAKT 520; Exakt GmbH, Norderstedt, Germany), and resin cured for one day. At the end of the curing, the specimens were attached to the slide by the Adhesive press system with the section of the part to be obtained by the EXAKT diamond cutting system (EXAKT 300 CP; Apparatebau GmbH, Norderstedt, Germany). The thickness of the final tissue sections was polished to $40 \pm 5 \mu \mathrm{m}$ using the EXAKT grinding system (KULZER EXAKT 400CS; EXAKT, Norderstedt, Germany). These tissues were stained for Goldner's trichrome.

The preserved amount of alveolar bone after extraction was measured by using a tissue sample slide of the undecalcified sample. The photographs taken at $\times 40$ / $\times 100$ magnification under the optical microscope were converted into jpeg files and the amount of alveolar bone regeneration was measured by Caseviewer (Version 2.0; 3D Histech, Budapest, Hungary). The histologic measurements were obtained by using 3 Histech company's Pannoramic 250 Flash III (No.3 Öv Street Budapest, Hungary) and digital images were obtained by Caseviewer. Bone volume measurement was based on blood vessels to the lingual and buccal alveolar ridge area.

\section{Statistical analysis}

Data were presented as mean and standard deviation (SD). The changes in gingival width were analyzed by IBM SPSS Statistics for Windows, Version 21.0 (IBM Co., Armonk, NY, USA). Changes in gingiva and bone volume between groups were compared using Mann-Whitney U-test followed by Kruskal-wallis test. A paired t-test was utilized to analyze time effect within groups. The $p$-values of $<0.05$ was considered as statistically significant. 


\section{Results}

\section{Clinical evaluations}

During the experimental period, all the experimental sites was healed uneventfully and no infection was observed. No other complications were observed.

Clinical observations showed that the width of gingiva at alveolar crest in each group decreased over time. There was no statistical difference in each group at 2 weeks (Fig. 2). There was statistically different in the control group and experimental groups at 4, 8, 12 weeks, respectively (Fig. $2, p<0.05$ ). The gingiva width of the surederm group was significantly higher than the control group at 6 weeks.

Within the group, the collatape group was significantly reduced at 4, 6, 8, 12 weeks compared to 2 weeks. The surederm group also showed decreased over time, but the difference was not significant. In the control group was much reduced at 4 weeks and then little more over time. The surederm group and the collatape group tended to decrease more over time. However, it was not statistically significant.

Gingival changes on buccal aspects showed a slight increase over time. The collatape group was statistical significantly smaller dimension compared with the control group at 2 weeks (Fig. 3, $p<0.05$ ). The surederm group showed greater compared with the control group and the collatape

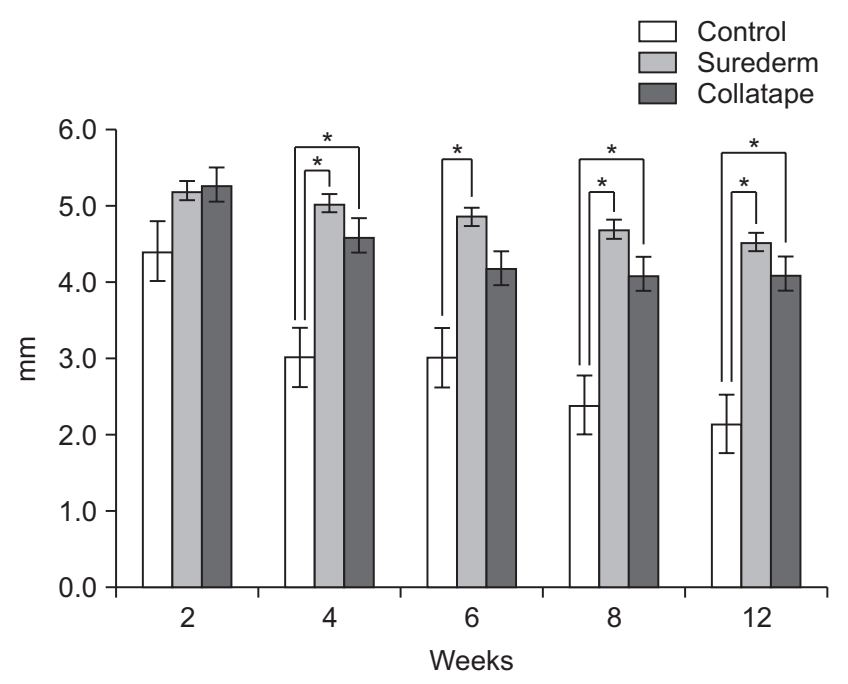

Fig. 2. The measurement of the gingiva at alveolar crest. ${ }^{*} p<0.05$. group at 4 weeks $(p<0.05)$. The both experimental groups showed significant differences compared to the control group at 6,12 weeks $(p<0.05)$. The surederm group was greater compared to the control group at 8 weeks $(p<0.05)$.

Within the group, experiment groups increased with time, but the differences were not significant. The control group was more reduced at 4 weeks and then decreased slightly over time. The surederm group increased to 4 weeks and then slightly decreased over time after 6 weeks. But it was not statistically significant. The collatape group appeared to increase slightly at 6 weeks, but tended to decrease. However, it was not statistically significant.

\section{Histological evaluations}

All groups were healed uneventfully after 12 weeks (Fig. 4). All barrier membranes were not exposed during the healing period. Fig. 5 showed a histological image of the control group. The extraction socket healed without intervention of epithelium basement membrane involved in keratinized epithelial and dermal layer were developed well (Fig. 5A). No periosteal membrane was observed in alveolar bone in contact with dermis (Fig. 5B). The upper and middle parts of the socket were not shaped like a socket and a new bone was observed (Fig. 5B, C). On the lower and lower parts of the socket, spaces were observed in the supporting bone of the alveolar bone containing blood vessels

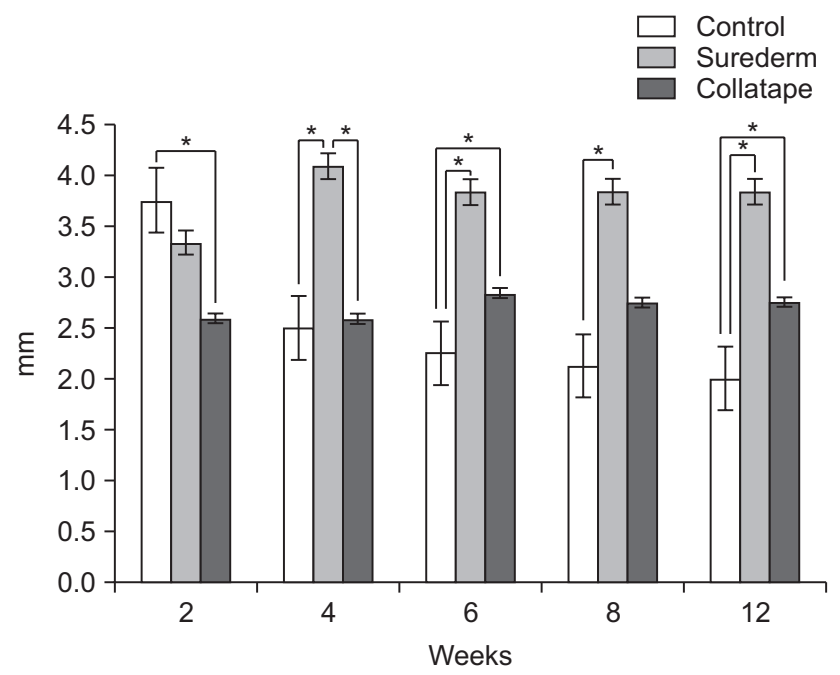

Fig. 3. The measurement of the gingiva at buccal side. ${ }^{*} p<0.05$. 

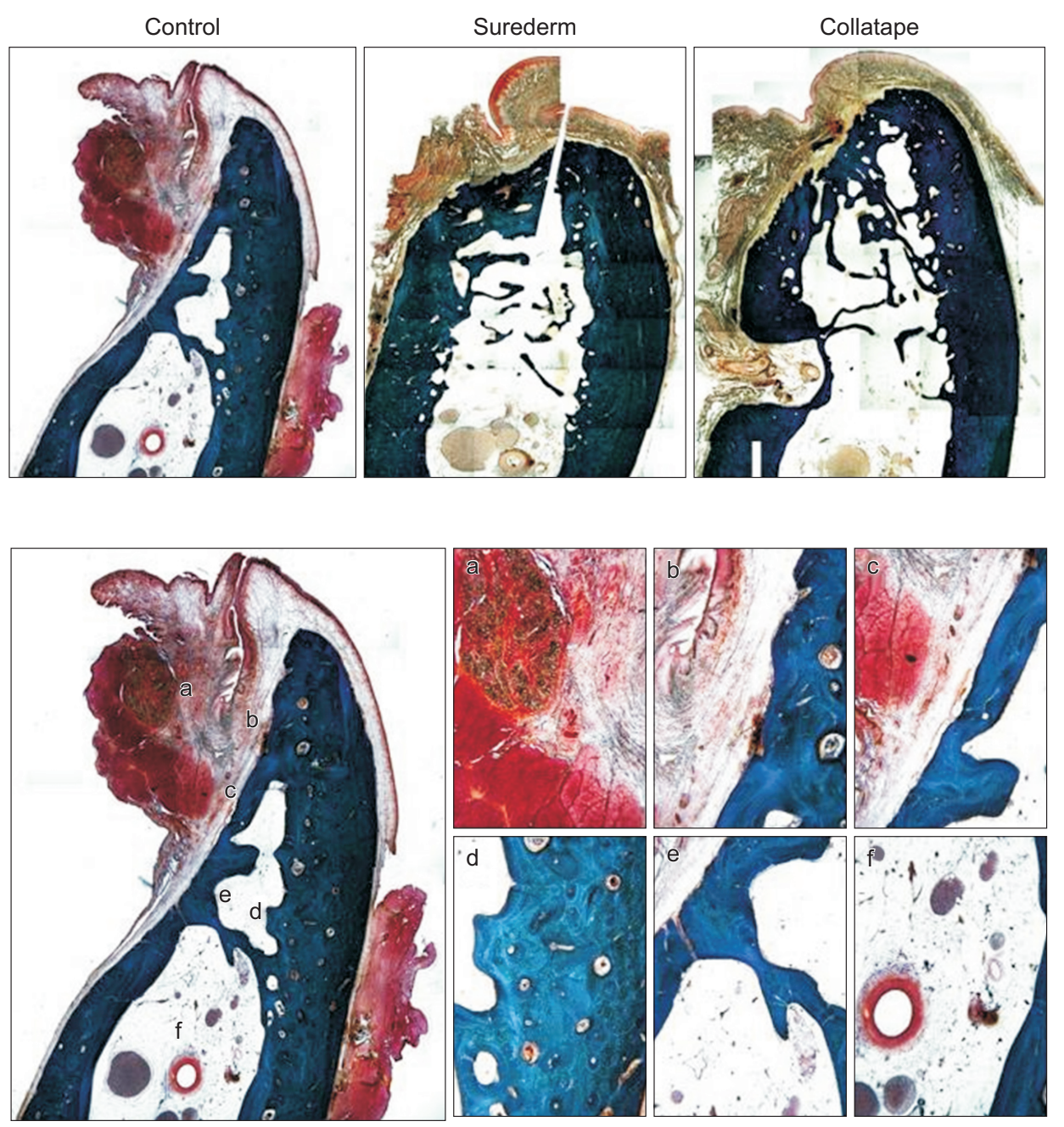

Fig. 4. The histological images for extraction socket after premolar extraction of the beagle dogs (Goldner's trichrome; $\times 40$ ).
(Fig. 5D, E). Adipose tissue cells in the bone marrow were located around nerve fiber bundles and blood vessels (Fig. 5E, F).

Fig. 6 shows a histological image of the surederm group. A well-healed keratinized epithelium and new bone deposits below the dermis were observed (Fig. 6A, B). Below it, a dark red-colored osteoid seam was observed around the alveolar bone (Fig. 6B, C). Osteoid seams were observed directly below and to the side and below the socket. There was a fatty tissue between them (Fig. 6D-F).

Fig. 7 shows a histological image of the collatape group. There was a fragment of the alveolar bone in the dermis tissue healed without epithelial involvement, and a new bone formation was observed downward (Fig. 7A, B). A connective tissue band was observed at the site of contact with the alveolar bone under the dermis, and an osteoid seam stained with a purplish red-colored purple dye was observed around the alveolar bone (Fig. 7B, C). Many osteoid seams were observed in the peripheral alveolar bone, just below the socket, laterally and downwardly. Between the two latters, there was a fatty tissue in the bone marrow (Fig. 7D-F).

The measured mean \pm SD alveolar bone volume was $25.36 \pm 2.73 \mathrm{~mm}^{2}$ in the control group, $42.66 \pm 1.52 \mathrm{~mm}^{2}$ in the surederm group, and $36.5 \pm 3.16 \mathrm{~mm}^{2}$ in the collatape group. Both experimental groups showed more alveolar bone volume than the control group. The statistically significant difference was observed between experimental groups and the control group (Fig. 8, $p<0.05$ ). Amount of alveolar bone volume in the surederm group had lager than the collatape group. The difference between the surederm group and the collatape group was statistically significant 

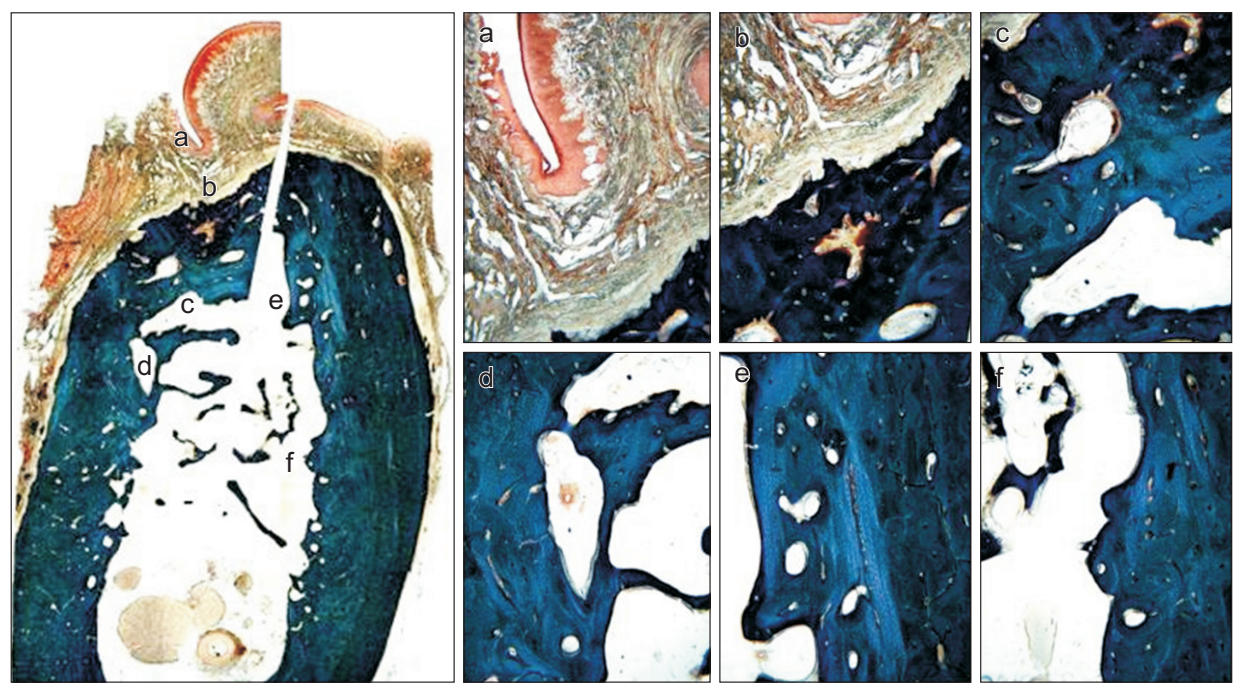

Fig. 6. The histological images for surederm treated alveolar socket after premolar extraction of the beagle dogs (Goldner's trichrome; left: $\times 40$; right: $\times 100)$.
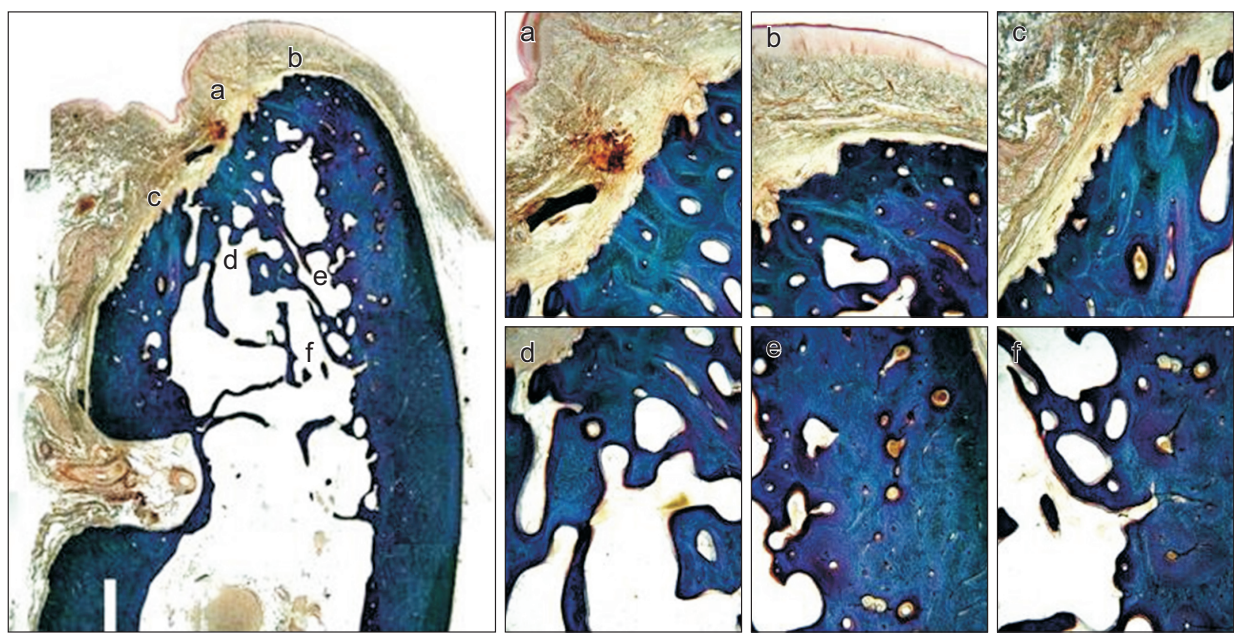

Fig. 7. The histological images for collatape treated alveolar socket after premolar extraction of the beagle dogs (Goldner's trichrome; left: $\times 40$; right: $\times 100)$.

(Fig. $8, p<0.05$ ).

\section{Discussion}

The lack of adequate keratinized mucosa around dental implants was associated with more plaque accumulation, tissue inflammation, mucosal recession as well as loss of attachment [21]. John et al. [22] studied ridge preservation with freeze-dried bone allograft and a collagen membrane compared to extraction alone. Collagen membrane group obtained a gingival thickness of $2.6 \mathrm{~mm}$, while gingival thickness of the extraction socket without any treatment was $2.1 \mathrm{~mm}$. On the buccal aspect, the change of soft tissue thickness was significantly different between the control group and ridge preservation groups. After extraction,

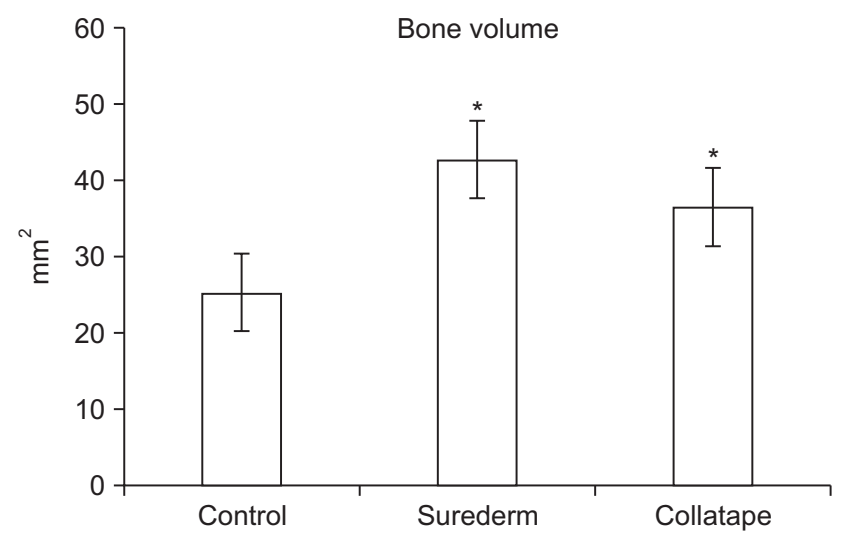

Fig. 8. The amount of alveolar bone after extraction was measured by using a tissue sample slide of the undecalcified sample $(p<0.05)$. Control: $\mathrm{n}=6$, Surederm: $\mathrm{n}=7$, Collatape: $\mathrm{n}=7 .{ }^{\star} p<0.05$. 
blood clots were formed in the alveolar bone, and tissues were formed in the order of granulation, provisional matrix, and woven bone. The mineralization was in progress around woven bone, and mature with a lamellar bone. Bone resorption was progressed in the lateral alveolar bone by the osteoclast, and bone resorption occurred more in the buccal crestal region than in the lingual bone with absorption of the bundle bone.

In one study, the mandibular pre-molars were extracted. After 8 weeks, one bone defect was surgically created bilaterally and the GBR was performed. The control group was treated with the bio-absorbable membrane of glycolide and lactide copolymer, and the experimental group was treated with acellular dermal matrix membrane [17]. The clinical comparison between the measurements of both groups showed an increase in the thickness of keratinized tissue and a reduction in the width of keratinized tissue over time. In comparison between the groups, the change in the amount and width of keratinized gingiva increased in the buccal side and decreased in the lingual side in the acellular dermal matrix membrane group than in the bio-absorbable membrane group at 16 weeks. Therefore, it was suggested that ridge preservation and soft tissue transplantation are necessary for the preservation of thickness and amount of soft tissue.

However, many studies have focused on the preservation of the alveolar bone in the extraction socket, and studies have been rare for the preservation and healing patterns of soft tissue on top of the alveolar bone. The quality and amount of bone is also important in implant treatment, but the amount and width of keratinized gingiva is more important to maintain long-term survival and success rate of implants. Thus, in this study, after removing teeth from beagle dogs, collagen membrane and acellular dermal matrix were applied to the upper part of the extraction sockets, and the healing patterns were observed in the gingiva and alveolar bone.

In this study, all groups were healed uneventfully. In the histological results of this study, after 12 weeks, the extraction sockets healed without intervention of epithelium basement membrane involved in keratinized epithelial and dermal layer were developed well. The experimental groups showed more new bone formation than the con- trol group. The surederm group showed that well-healed keratinized epithelium and new bone deposits below the dermis were observed. A dark red-colored osteoid seam was observed around the alveolar bone. Lekovic et al. [23] used an occlusive membrane for preventing soft tissue and epithelial migration into the healing socket. A secondary benefit to the use of an occlusive membrane was that there appeared to be less external ridge resorption in the early healing phase, although the exact mechanism of this effect remains unclear. Barone et al. [24] reported soft tissue and hard tissue changes in bone graft and membrane cover after extraction. Comparison of clinical variables at 4 months showed that the width of keratinized gingiva was observed in the grafted sites when compared to non-grafted sites. In the non-grafted group, horizontal bone resorption was significantly higher for those sites which had buccal bone thickness of $1 \mathrm{~mm}$ when compared to sites which had a buccal bone thickness of $3 \mathrm{~mm}$.

In this study, clinical observations showed that the width of gingiva at alveolar crest in each group decreased over time. There was no statistical difference in each group at 2 weeks. There was statistically different in the control group and experimental groups at 4, 8, 12 weeks, respectively. The gingival width of the surederm group was significantly higher than the control group at 6 weeks.

Within the group, the collatape group decreased significantly over other weeks compared to 2 weeks. The surederm group also showed decreased over time, but the difference was not significant. In the control group was much reduced at four weeks and then decreased slightly more over time. The surederm group and the collatape group tended to decrease over time. However, it was not statistically significant. Changes in gingiva on buccal aspects showed a slight increase over time. The collatape group was statistical significantly smaller dimension compared with the control group at 2 weeks. The surederm group showed greater compared with the control group and the collatape group at 4 weeks. The experimental groups showed statistical significantly compared with the control group at 6,12 weeks. The surederm group was greater compared with the control group at 8 weeks. Within the group, experiment groups increased with time, but the difference was not significant. The control group was much 
reduced in the four weeks and then decreased slightly over time. The surederm group was increased at four weeks, although decreasing slightly over time from 6 weeks. But it was not statistically significant. The collatape group showed a little increase at 6 weeks, tended to decrease, but it was not statistically significant. This results also showed that the amount of gingiva was increased in comparison with those using soft tissue or membrane in the extraction socket, especially in the surederm group than in the collatape group. When the acellular dermal matrix was applied to the top of the extraction socket, it was well matched to the surrounding soft tissue to prevent epithelial or soft tissue penetration into the extraction socket. It was presumed that it was absorbed slowly relative to the collagen membrane and helped to form keratinized gingiva. Acellular dermal matrix has a three-dimensional dermal structure. By removing the elements that cause the immune response, the dermis without immunologic response provides a framework for the inflow of fibroblasts, the generation of nerves, and the regeneration of blood vessels. In addition, formation of the epithelial layer due to the entry of keratinocytes and introduction of fibroblasts make it easy to engraft into the selfdermal layer since the basement membrane structure is preserve.

Heinemann et al. [25] reported that there was a significant difference in bone level changes in the group using Bio-Oss Collagen compared with extraction socket group. Another study showed that ridge loss was similar regardless of bone graft, and thicker buccal plate was associated with less ridge loss in the vertical dimension, in addition no significant difference in the percentage of new bone formation [26]. In this study, a collagen membrane and acellular dermal matrix were applied to the top of the extraction socket. The bone graft was not applied because it could affect healing of extraction sockets or changes in bone volume. Both experimental groups showed more alveolar bone volume than the control group. The statistically significant difference was observed between experimental groups and the control group. This study also showed more bone volume in the acellular dermal matrix group. Similar to other studies, acellular dermal matrix had shown better results because it rapidly bound to the soft tissue at the top of the extraction socket rapidly, prevented intrusion of epithelial and soft tissue into the extraction socket, and preserved blood clots in the extraction socket, that did not interfere with healing of bone tissue [27,28]. Barone et al. [29] investigated 40 patients receiving treatment at the hospital for one year. Histological evaluation of the cells after extraction-alone sites showed large marrow spaces filled with large numbers of adipocytes with only scarce inflammatory cells. A lamellar bone was also present within the bone marrow. Protection of a blood clot in the defect and exclusion of gingival connective tissue and provides covert space for osteoblasts in bone to migrate, which is critical to success [30]. The results showed that the collagen sponge prevents soft tissue from infiltrating into the lower region [31]. In the histological results of this study, all groups were healed uneventfully after 12 weeks. The extraction socket healed without intervention of epithelium basement membrane involved in keratinized epithelial and dermal layer were developed well. In the control group, no periosteal membrane was observed in alveolar bone in contact with dermis. The upper and middle parts of the socket were not shaped like a socket and a new bone was observed. In the study of Barone et al. [29], lamellar bone was also present within the bone marrow. Similar to our histological results, the extraction socket showed a typical trabecular bone pattern, and many adipocytes were filled with less inflammatory cells. In the surederm and the collatape groups, the connective tissue band was observed at the site of contact with the alveolar bone under the dermis, and an osteoid seam stained with a purplish red-colored purple dye was observed around the alveolar bone. Many osteoid seams were observed in the peripheral alveolar bone, just below the socket, laterally and downwardly. Between the two latters, there was a fatty tissue in the bone marrow.

In summary, clinically, the soft tissue changes were decreased in the alveolar crest in all groups, and in the buccal side, the control group was decreased and the experimental groups were increased. Histological by the area of the extraction socket, experimental groups were significantly greater than the control group. Thus, the socket was covered with soft tissue, this study showed not only increased the amount and width of gingiva, but also helped maintain the volume of the lower bone tissue. However, it did not promote bone healing. In the future, it will be necessary 
to increase the amount and width of gingiva during ridge preservation and to develop the methods of promoting healing of bone tissue including bone graft containing growth factors.

\section{Conflicts of Interest}

The authors declare that they have no competing interests.

\section{ORCID}

\author{
Xing-Hui Piao \\ https://orcid.org/0000-0003-4497-4977 \\ Hyo-Seon Park \\ https://orcid.org/0000-0002-5259-4344 \\ Eui-Ri Na \\ https://orcid.org/0000-0002-1584-8966 \\ Young-Joon Kim \\ https://orcid.org/0000-0003-1397-8166 \\ Jong-Wook Moon \\ https://orcid.org/0000-0002-6688-0210
}

\section{References}

1. Araújo MG, Lindhe J. Dimensional ridge alterations following tooth extraction. An experimental study in the dog. J Clin Periodontol 2005;32:212-218. doi: 10.1111/j.1600051X.2005.00642.x.

2. Araújo MG, Lindhe J. Ridge alterations following tooth extraction with and without flap elevation: an experimental study in the dog. Clin Oral Implants Res 2009;20:545-549. doi: 10.1111/j.1600-0501.2008.01703.x.

3. Pagni G, Pellegrini G, Giannobile WV, Rasperini G. Postextraction alveolar ridge preservation: biological basis and treatments. Int J Dent 2012;2012:151030. doi: 10.1155/2012/151030.

4. Fickl S, Zuhr O, Wachtel H, Bolz W, Huerzeler MB. Hard tissue alterations after socket preservation: an experimental study in the beagle dog. Clin Oral Implants Res 2008;19:1111-1118. doi: 10.1111/j.1600-0501.2008. 01575.x.

5. Araújo M, Linder E, Wennström J, Lindhe J. The influence of Bio-Oss Collagen on healing of an extraction socket: an experimental study in the dog. Int J Periodontics Restorative Dent 2008;28:123-135.

6. Evian CI, Cutler S. Autogenous gingival grafts as epithelial barriers for immediate implants: case reports. J Periodontol
1994;65:201-210. doi: 10.1902/jop.1994.65.3.201.

7. Vieira Ede O, Fidel Junior RA, Figueredo CM, Fischer RG. Clinical evaluation of a dermic allograft in procedures to increase attached gingiva width. Braz Dent J 2009;20:191194. doi: 10.1590/s0103-64402009000300003.

8. Cummings LC, Kaldahl WB, Allen EP. Histologic evaluation of autogenous connective tissue and acellular dermal matrix grafts in humans. J Periodontol 2005;76:178-186. doi: 10.1902/jop.2005.76.2.178.

9. Lee CW, Yeo HH, Son BH. An experimental study of autogenous mucosa and AlloDerm grafts in rabbits. Oral Biol Res 1999;23:169-198.

10. Tal H, Moses O, Zohar R, Meir H, Nemcovsky C. Root coverage of advanced gingival recession: a comparative study between acellular dermal matrix allograft and subepithelial connective tissue grafts. J Periodontol 2002;73:1405-1411. doi: 10.1902/jop.2002.73.12.1405.

11. Kim MJ, Chung HJ, Kim OS, Kim YJ. Histological healing after grafting of bilayer artificial dermis in the oral environment. J Korean Acad Periodontol 2003;33:289-299. doi: 10.5051/jkape.2003.33.2.289.

12. Hirsch A, Goldstein M, Goultschin J, Boyan BD, Schwartz Z. A 2-year follow-up of root coverage using sub-pedicle acellular dermal matrix allografts and subepithelial connective tissue autografts. J Periodontol 2005;76:13231328. doi: 10.1902/jop.2005.76.8.1323.

13. Aichelmann-Reidy ME, Yukna RA, Evans GH, Nasr HF, Mayer ET. Clinical evaluation of acellular allograft dermis for the treatment of human gingival recession. J Periodontol 2001;72:998-1005. doi: 10.1902/jop.2001.72.8.998.

14. Wei PC, Laurell L, Lingen MW, Geivelis M. Acellular dermal matrix allografts to achieve increased attached gingiva. Part 2. A histological comparative study. J Periodontol 2002;73:257-265. doi: 10.1902/jop.2002.73.3.257.

15. Santos A, Goumenos G, Pascual A. Management of gingival recession by the use of an acellular dermal graft material: a 12-case series. J Periodontol 2005;76:1982-1990. doi: 10.1902/jop.2005.76.11.1982.

16. Sudarsan S, Arun KV, Priya MS, Arun R. Clinical and histological evaluation of alloderm GBR and BioOss in the treatment of Siebert's class I ridge deficiency. J Indian Soc Periodontol 2008;12:73-78. doi: 10.4103/0972-124X.44099.

17. Borges GJ, Novaes AB Jr, Grisi MF, Palioto DB, Taba M Jr, de Souza SL. Acellular dermal matrix as a barrier in guided bone regeneration: a clinical, radiographic and histomorphometric study in dogs. Clin Oral Implants Res 2009;20:1105-1115. doi: 10.1111/j.16000501.2009.01731.x.

18. Luczyszyn SM, Papalexiou V, Novaes AB Jr, Grisi MF, Souza SL, Taba M Jr. Acellular dermal matrix and hydroxyapatite in prevention of ridge deformities after tooth extraction. Implant Dent 2005;14:176-184. doi: 10.1097/01. id.0000165082.77499.41. 
19. Fotek PD, Neiva RF, Wang HL. Comparison of dermal matrix and polytetrafluoroethylene membrane for socket bone augmentation: a clinical and histologic study. J Periodontol 2009;80:776-785. doi: 10.1902/jop.2009.080514.

20. Fowler EB, Breault LG, Rebitski G. Ridge preservation utilizing an acellular dermal allograft and demineralized freeze-dried bone allograft: Part II. Immediate endosseous implant placement. J Periodontol 2000;71:1360-1364. doi: 10.1902/jop.2000.71.8.1360.

21. Lin GH, Chan HL, Wang HL. The significance of keratinized mucosa on implant health: a systematic review. J Periodontol 2013;84:1755-1767. doi: 10.1902/jop.2013.120688.

22. Iasella JM, Greenwell H, Miller RL, Hill M, Drisko C, Bohra AA, Scheetz JP. Ridge preservation with freeze-dried bone allograft and a collagen membrane compared to extraction alone for implant site development: a clinical and histologic study in humans. J Periodontol 2003;74:990-999. doi: 10.1902/jop.2003.74.7.990.

23. Lekovic V, Camargo PM, Klokkevold PR, Weinlaender M, Kenney EB, Dimitrijevic B, Nedic M. Preservation of alveolar bone in extraction sockets using bioabsorbable membranes. J Periodontol 1998;69:1044-1049. doi: 10.1902/ jop.1998.69.9.1044.

24. Barone A, Ricci M, Tonelli P, Santini S, Covani U. Tissue changes of extraction sockets in humans: a comparison of spontaneous healing vs. ridge preservation with secondary soft tissue healing. Clin Oral Implants Res 2013;24:12311237. doi: 10.1111/j.1600-0501.2012.02535.x.

25. Heinemann F, Hasan I, Schwahn C, Bourauel C, Mundt T. Bone level change of extraction sockets with Bio-Oss col- lagen and implant placement: a clinical study. Ann Anat 2012;194:508-512. doi: 10.1016/j.aanat.2011.11.012.

26. Brownfield LA, Weltman RL. Ridge preservation with or without an osteoinductive allograft: a clinical, radiographic, micro-computed tomography, and histologic study evaluating dimensional changes and new bone formation of the alveolar ridge. J Periodontol 2012;83:581-589. doi: 10.1902/jop.2011.110365.

27. Hu XL, Lin Y, Wang J, Wu H, Qiu LX, Zhang Y. [Clinical study of tissue preservation of extraction socket with BioOss collagen and acellular dermal matrix]. Zhonghua Kou Qiang Yi Xue Za Zhi 2009;44:513-516. Chinese.

28. Novaes AB Jr, Souza SL. Acellular dermal matrix graft as a membrane for guided bone regeneration: a case report. Implant Dent 2001;10:192-196. doi: 10.1097/00008505200107000-00009.

29. Barone A, Aldini NN, Fini M, Giardino R, Calvo Guirado JL, Covani U. Xenograft versus extraction alone for ridge preservation after tooth removal: a clinical and histomorphometric study. J Periodontol 2008;79:1370-1377. doi: 10.1902/jop.2008.070628.

30. Lang NP, Hämmerle CH, Brägger U, Lehmann B, Nyman SR. Guided tissue regeneration in jawbone defects prior to implant placement. Clin Oral Implants Res 1994;5:92-97. doi: 10.1034/j.1600-0501.1994.050205.X.

31. Kim YK, Yun PY, Lee HJ, Ahn JY, Kim SG. Ridge preservation of the molar extraction socket using collagen sponge and xenogeneic bone grafts. Implant Dent 2011;20:267272. doi: 10.1097/ID.0b013e3182166afc. 